\title{
Efeitos da exposição a estímulos aversivos e apetitivos incontroláveis sobre o comportamento verbal em contingências de reforço positivo
}

\author{
Effects of exposure to uncontrollable, aversive and \\ appetitive stimuli on verbal behavior under \\ positive reinforcement contingencies
}

\author{
Tatiany Honório PORTO \\ Maria Beatriz Barreto do CARMO' \\ Reginaldo do Carmo AGUIAR \\ Vanessa PENNA-GONÇALVES ${ }^{2}$ \\ Gerson Yukio TOMANARI ${ }^{3}$
}

\begin{abstract}
Resumo
Foram analisados efeitos de diferentes histórias de incontrolabilidade por perda ou ganho de pontos sobre o desempenho posterior de participantes humanos na construção de frases. Inicialmente, os participantes podiam ganhar ou perder pontos independentemente de qualquer característica da frase construída. Posteriormente, recebiam pontos por construir frases iniciadas apenas pelo pronome "ele". Os resultados mostram que a exposição à incontrolabilidade pode dificultar condições posteriores de novas aprendizagens sob reforçamento positivo. Interessantemente, essas dificuldades foram menos acentuadas e, em certos casos, até mesmo superadas, no caso de uma história de exposição a ganhos incontroláveis de pontos. Em contrapartida, no caso de uma história de perdas incontroláveis de pontos, aprendizagens subsequentes sob reforço positivo tenderam a ser prejudicadas. Esses resultados contribuem para os estudos de incontrolabilidade e desamparo aprendido, em particular por apresentar alternativas metodológicas passíveis de aplicação a respostas verbais em humanos.
\end{abstract}

Unitermos: Comportamento verbal. Desamparo aprendido. História comportamental. Incontrolabilidade.

\begin{abstract}
Human participants were exposed to a task in which, by constructing sentences, the effects of different stories of uncontrollability were assessed through the loss or acquisition of points in a subsequent exposure to a new learning condition. Initially, subjects could lose or win points regardless of any specific response. Subsequently, they would receive points for constructing any sentence beginning with the pronoun "he." Results show that exposure to uncontrollability can make it difficult to acquire a subsequent learning task. In particular, the effects of the history of exposure to uncontrollable points gains were relatively short-lived, concentrated at the beginning of the learning process and

$\cot$

1 Doutorandos, Universidade de São Paulo, Instituto de Psicologia, Programa de Pós-Graduação em Psicologia. São Paulo, SP, Brasil.

2 Mestranda, Universidade de São Paulo, Instituto de Psicologia, Programa de Pós-Graduação em Psicologia. São Paulo, SP, Brasil.

3 Universidade de São Paulo, Instituto de Psicologia, Departamento de Psicologia Experimental. Av. Professor Mello Moraes, 1721, 05508-030, Butantã, SP, Brasil. Correspondência para/Correspondence to: G.Y. TOMANARI. E-mail: <tomanari@usp.br>.
\end{abstract}


tending to recover throughout the exposure to the new task. On the other hand, the effects of uncontrollability through the loss of points had a detrimental effect on the acquisition of the new task. These results contribute to the study of uncontrollability and learned helplessness, particularly by proposing alternative methods using human verbal responses.

Uniterms: Verbal behavior. Learned helplessness. Behavioral history. Uncontrollability.

Na perspectiva analítico-comportamental, o controle de uma resposta por um estímulo consequente pode ser conceituado como relações de probabilidade entre eventos e expresso pela notação $\mathrm{p}(S / R) \neq \mathrm{p}(S / n R)$, em que p designa a probabilidade de ocorrência de um estímulo (S) após a emissão de uma resposta (R). Quando a probabilidade de S, após a emissão de R, é diferente da probabilidade de $S$, na ausência de R (nR), se diz que há uma relação de controle de $\mathrm{R}$ por $\mathrm{S}$. Por outro lado, quando a probabilidade de $S$, dado $R$, é igual à probabilidade de $S$, na ausência de $R$, isto é, $p(S / R)=p(S / n R)$, tem-se uma condição de incontrolabilidade (Hunziker, 2003).

Estudos experimentais têm demonstrado que, no desenvolvimento de repertórios comportamentais, um processo de aprendizagem pode ser substancialmente afetado por uma história de incontrolabilidade a estímulos aversivos. Por exemplo, um organismo exposto a estímulos aversivos sobre os quais não exerce qualquer controle pode apresentar dificuldades futuras de eliminar ou evitar estímulos aversivos, ainda que esteja sob condições passíveis de controle. Dificuldades de fuga ou esquiva de estímulos aversivos em decorrência de uma história de incontrolabilidade também aversiva é conhecida por desamparo aprendido (Maier \& Seligman, 1976). Trata-se de um fenômeno já observado em várias espécies, entre as quais a de ratos (Maier, Albin \& Testa, 1973; Seligman, Rosellini \& Kozak, 1975), cães (Overmier \& Seligman, 1967), baratas (Brown, Howe \& Jones, 1990) e humanos (Hiroto \& Seligman, 1975; Matute, 1994; Peterson, Maier \& Seligman, 1993).

A partir do paradigma metodológico clássico do desamparo aprendido (Maier \& Seligman, 1976), a literatura tem buscado analisar as variáveis que modulam esse processo, tanto no momento prévio de incontrolabilidade, quanto na ocasião em que novas aprendizagens deveriam ocorrer.

No que diz respeito à história de incontrolabilidade, em particular, estudos têm procurado isolá-la da natureza do estímulo (apetitivo vs aversivo) e do tipo

338 de estímulo (choques elétricos, jatos de ar, entre outros).
Em razão de uma confluência de variáveis, entretanto, não se pode afirmar em que medida o desamparo aprendido é um efeito dessas variáveis (natureza ou tipo de estímulo, por exemplo) ou, preponderantemente, da incontrolabilidade em si. Nesse segundo caso, abrir-se-ia a possibilidade de se verificar desamparo aprendido também em função de uma história de incontrolabilidade a estímulos apetitivos.

Capelari e Hunziker (2005) utilizaram um procedimento análogo ao tradicionalmente utilizado no estudo do desamparo com ratos, mas substituíram os estímulos aversivos incontroláveis por estímulos apetitivos incontroláveis. Seus resultados mostraram que a história com estímulos apetitivos incontroláveis não afetou a aprendizagem posterior do comportamento de fuga, achados corroborados por alguns estudos (Beatty \& Maki, 1979; Calef et al.,1984), mas não por outros (Caspy \& Lubow, 1981; Enberg, Welker, Hansen \& Thomas, 1972; Ferrándiz \&Vicente, 1997; Job, 1987; Sonoda \& Hirai, 1992; Welker, 1976; Wheatley, Welker \& Miles, 1977).

Do mesmo modo como a incontrolabilidade não se encontra suficientemente isolada da natureza do estímulo incontrolável, o papel do uso de choques elétricos no desamparo aprendido também carece de especificação. A maioria dos estudos na área, com exceção aos que empregaram sujeitos humanos, utilizou choque elétrico como estímulo aversivo incontrolável. Portanto, a ocorrência de desamparo aprendido, a partir de uma história com outros tipos de estímulos aversivos incontroláveis, também é um ponto a ser analisado, tal como propõem Carvalho Neto, Maestri e Menezes (2007), ao empregarem jatos de ar quente como estímulo aversivo em ratos.

Na análise do desamparo aprendido, a contingência de reforço vigente no momento de controlabilidade é um parâmetro igualmente relevante. Originalmente, o desamparo aprendido foi definido como sendo uma dificuldade de aprendizagem em contingências de reforço negativo, fuga e esquiva. No entanto, pesquisadores têm questionado a generalidade do fenômeno, 
que possivelmente poderia ser também observado em contingências de reforço positivo (Catania, 1999, para um detalhamento introdutório sobre contingências de reforço), conforme dados da literatura têm indicado (Calef, Choban, Shaver, Dye \& Geller, 1986; Caspy \& Lubow, 1981; Rosellini, 1978; Rosellini \& DeCola, 1981; Rosellini, DeCola \& Shapiro, 1982). No entanto, esses resultados não estão imunes a controvérsias, pois Chen e Amsel (1977) não obtiveram dados na mesma direção.

A partir da revisão acima, portanto, é evidente a necessidade de se identificarem com maior precisão os fatores que levam ao desamparo aprendido. Para isso, alternativas metodológicas, possivelmente distantes do paradigma clássico relatado por Maier e Seligman (1976), devem ser exploradas. Por essa perspectiva, o presente estudo analisou o desamparo aprendido em participantes humanos expostos a uma tarefa de construção de frases, na qual respostas verbais compõem contingências de reforço positivo, reforço negativo ou punição, e têm ganho ou perda de pontos como consequência (Hamasaki \& Tomanari, 2007, 2009; Tomanari, Carvalho, Góes, Lira \& Viana, 2007).

No presente estudo, os participantes foram expostos a uma condição experimental em que recebiam pontos por construir frases iniciadas pelo pronome "ele", mas não por frases iniciadas com qualquer outro pronome. O desempenho nessa tarefa, avaliado pela frequência de uso do pronome "ele", foi analisado em função de duas condições antecedentes de incontrolabilidade: ganho ou perda de pontos independentemente de qualquer característica da frase construída. Para fins de controle, um grupo de participantes não passou pela história de incontrolabilidade.

Essa proposta metodológica visou avaliar se as histórias de incontrolabilidade afetariam o desempenho posterior na tarefa de construção de frases com ganho controlável de pontos, bem como se esse desempenho seria diferentemente afetado a depender do tipo de incontrolabilidade prévia, apetitiva ou aversiva.

\section{Método}

\section{Participantes}

Voluntariaram-se ao estudo dez participantes, de ambos os sexos, dentre os quais se encontravam estudantes universitários de diversos cursos de graduação, excluindo-se o de psicologia.

\section{Instrumentos}

Para coleta de dados, foram utilizados microcomputadores do tipo PC, equipados com monitores coloridos de 15 polegadas e mouse, nos quais estava instalado o aplicativo Verbal 2.51, configurado para executar o procedimento descrito a seguir e para registrar as respostas dos participantes (Tomanari, Pavão \& Benassi, 2003).

\section{Procedimentos}

Os participantes foram submetidos a uma tarefa de construção de frases e instruídos conforme descrito em Tomanari et al. (2007). Verbos na forma infinitiva foram apresentados, sequencialmente, na parte centro-superior do monitor de vídeo. Tratava-se de verbos regulares, concretos e abstratos, da primeira e da segunda conjugação. Escolhidos entre os usados cotidianamente, foram originalmente propostos por Matos, Cirino, Passos, Damiani e Frochtengarten (1995), e encontram-se listados em Matos e Tomanari (2002).

A apresentação dos verbos era acompanhada, simultaneamente, por três menus drop-down, alinhados horizontalmente no centro do monitor. Para construir uma frase, o participante devia selecionar, consecutivamente, uma dentre seis opções disponíveis em três menus:

1) Pronome: O participante escolhia, no primeiro menu, um dentre os seis pronomes do caso reto para iniciar sua frase [eu, tu, ele(a), nós, vós, eles(as)].

2) Verbo: $O$ segundo menu apresentava um mesmo verbo conjugado em seis diferentes tempos verbais (presente, pretérito perfeito, pretérito imperfeito, pretérito mais-que-perfeito, futuro do presente e futuro do pretérito) e em acordo com o pronome selecionado pelo participante no menu anterior.

3) Complemento: Para finalizar a frase, o participante dispunha, no terceiro menu, de cinco opções de complementos verbais, entre diferentes advérbios e locuções adverbiais (como, por exemplo, com carinho, muito, realmente, flores, bem, entre outras locuções 
adverbiais), além da opção sempre disponível de não utilizar um complemento (opção"sem complemento") para o caso de verbos intransitivos, por exemplo.

Os menus eram ativados por um único acionamento do botão do mouse sobre cada um deles, e, havendo a seleção de um dos seus itens, esse menu era desativado e o seguinte imediatamente ativado; assim, uma vez feita uma seleção, não era possível alterá-la posteriormente. Após a seleção final no menu de complementos, com base em instruções do experimentador, o participante devia ler em voz alta a frase construída e, na sequência, acionar com o mouse o botão contendo a palavra "OK", situado na parte inferior da tela. Inicialmente, um contador de pontos localizado na parte superior central do monitor indicava 1000 pontos. Imediatamente, após o encerramento da frase, seguiam-se diferentes consequências em função do pronome selecionado pelo participante e da contingência de reforço em vigor (ver detalhamento adiante). Entre o encerramento de uma frase e o início da seguinte havia sempre um intervalo de 1,5s, durante o qual a tela permanecia completamente branca e inativa. A cada frase, uma nova sequência dos elementos que compunham os menus era sorteada, garantindo-se a equiprobabilidade de repetições de um mesmo pronome ou tempo verbal em uma mesma posição na lista.

O experimento foi composto por duas fases. A primeira visou construir uma história experimental de incontrolabilidade por exposição a ganhos (Participantes P1, P8, P9 e P10) e perdas (Participantes P11, P12 e P13) de pontos, independentemente de qualquer resposta emitida ao longo da construção de 18 frases. No primeiro caso, a construção de cada frase era seguida pela apresentação do número "+10" no centro da tela, em referência aos pontos que eram imediatamente adicionados no contador. No segundo caso, era seguida de"-10", quantidade de pontos imediatamente subtraída no contador. Dessa primeira fase, não fizeram parte P22, P27 e P28, cujas participações tiveram início na fase seguinte, descrita abaixo.

Na segunda fase, todos os dez participantes construíram 48 novas frases. Nesse momento, apenas a escolha do pronome "ele" era sempre seguida pela apresentação do número "+10" no centro da tela e do acréscimo de 10 pontos no contador. A escolha de qualquer um dos outros cinco pronomes era seguida por"0"(Tabela 1).

Essa pesquisa foi conduzida em acordo com as diretrizes do Conselho Nacional de Ética em Pesquisa por meio da Resolução CNS 196/96. Os participantes assinaram o Termo de Consentimento Livre e Esclarecido acerca de sua participação voluntária e foram enfaticamente informados de que poderiam deixar o experimento a qualquer momento.

\section{Resultados}

A Figura 1 mostra o número médio de escolhas do pronome "ele" na construção das 48 frases, por cada um dos dez participantes, ao longo de blocos de seis frases. Para os sete participantes que passaram por exposição incontrolável de ganho e perda de pontos, são mostradas as 18 primeiras frases iniciais, também no decorrer de blocos de seis frases.

Ao longo da construção de frases acompanhadas por eventos incontroláveis, os participantes que ganharam e perderam pontos escolheram o pronome "ele" aproximadamente uma vez em cada bloco de seis frases. Considerando-se haver seis diferentes pronomes disponíveis, as escolhas dos participantes não demonstravam padrões ou preferências sistemáticas (Figura 1).

No que diz respeito à construção das 48 frases sob reforço positivo, os dados mostram que todos os

\begin{tabular}{|c|c|c|c|c|}
\hline \multirow{2}{*}{ Participantes } & \multirow{2}{*}{\multicolumn{2}{|c|}{ História de incontrolabilidade (18 frases) }} & \multicolumn{2}{|c|}{ Contingência de Reforço Positivo (48 frases) } \\
\hline & & & Consequência ao uso de "ele" & Consequência ao uso de outros pronomes \\
\hline P1, P8, P9, P10 & Ganho de pontos & +10 & & \\
\hline $\mathrm{P} 11, \mathrm{P} 12, \mathrm{P} 13$ & Perda de pontos & -10 & +10 & 0 \\
\hline P22, P27, P28 & - & & & \\
\hline
\end{tabular}


Ref. Positivo

Sem incontrolabilidade

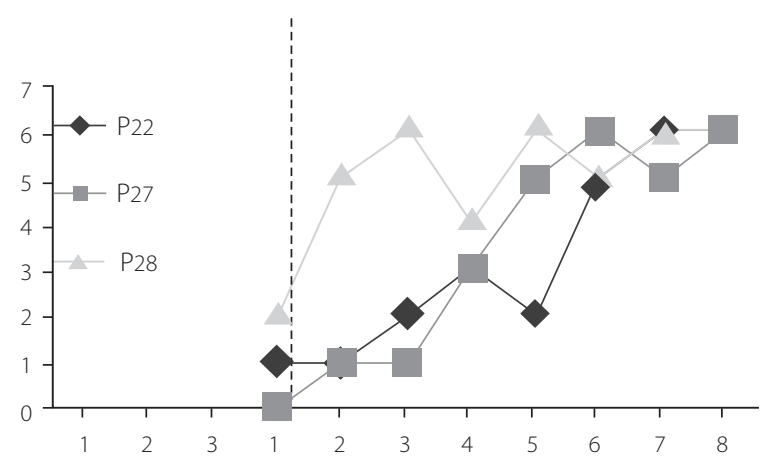

Incontrolabilidade por perda

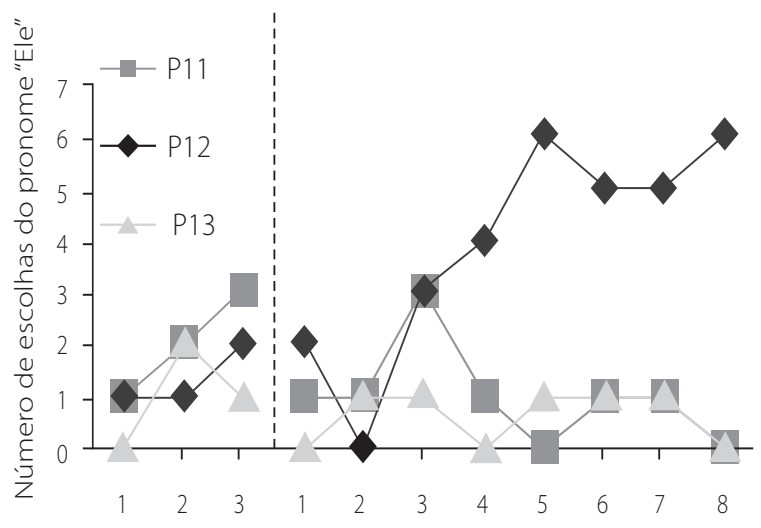

Incontrolabilidade por ganho

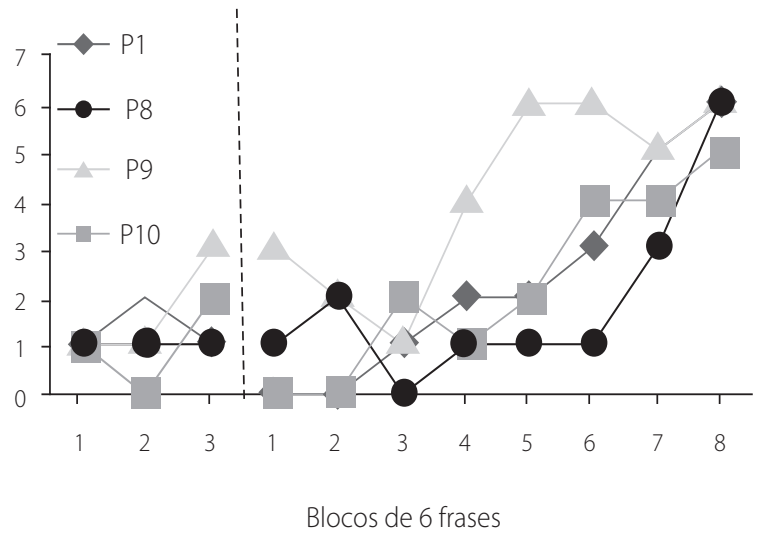

Figura 1. Número médio de escolhas do pronome "ele" na construção das 48 frases (Ref. Positivo), por cada um dos dez participantes, ao longo de blocos de seis frases. Para os sete participantes que passaram por exposição incontrolável de ganho e perda de pontos, são mostradas as 18 primeiras frases iniciais, também no decorrer de blocos de seis frases (INC). São Paulo (SP), 2009.

Nota: INC: condição de incontrolabilidade. participantes que não passaram pela história de exposição a ganhos ou perdas incontroláveis aumentaram gradual e sistematicamente a frequência de uso do pronome "ele". No primeiro bloco, os três participantes empregaram o pronome "ele" entre zero e duas vezes. No oitavo e último bloco, esses participantes utilizaram o pronome "ele" seis vezes, ou seja, em todas as frases que construíram. Essas curvas de aprendizagem podem agora ser comparadas com os participantes que passaram pela história de ganhos e perdas incontroláveis.

Os participantes que foram expostos a ganhos incontroláveis de pontos, à semelhança dos anteriormente descritos, aumentaram a frequência de escolha do pronome "ele" ao longo dos blocos de frases. No entanto, diferentemente dos anteriores, houve uma tendência de diminuição inicial, nos dois primeiros blocos, antes que o aumento gradual e sistemático passasse a ocorrer do quarto ao oitavo bloco. No oitavo bloco, todos os participantes usaram o pronome "ele" em praticamente todas as frases construídas.

Por fim, diferentemente dos participantes que não foram expostos a história de incontrolabilidade ou foram expostos a ganhos incontroláveis, os participantes expostos a perdas incontroláveis de pontos tenderam a não empregar o pronome "ele", cujo uso estava sob contingência de reforço positivo. Dois participantes, P12 eP13, diminuíram o uso do pronome"ele"em relação às 18 frases iniciais ao longo dos oito blocos de frases. Esses dois participantes responderam aleatoriamente entre todos os outros pronomes disponíveis, não predominando a escolha de nenhum pronome. Um único participante, P11, respondeu de modo semelhante aos participantes expostos a ganhos incontroláveis, isto é, após uma diminuição inicial, a frequência de uso do pronome "ele" aumentou até tornar-se exclusiva no oitavo bloco.

\section{Discussão}

Os resultados mostram que a exposição a ganhos ou perdas incontroláveis de pontos produziu efeitos no processo subsequente de reforço positivo pelo uso do pronome"ele". Comparados aos participantes que não passaram por condições iniciais incontroláveis, a exposição a eventos incontroláveis, tanto de ganhos quanto de perdas, tendeu a reduzir a frequência de 
reforços obtidos. Interessantemente, esses efeitos foram distintos a depender de a história de exposição a eventos incontroláveis ter-se dado por perda ou por ganho de pontos.

Para os participantes expostos à história de ganhos incontroláveis, a redução na frequência da resposta reforçada foi relativamente efêmera, concentrada no início da nova fase de aprendizagem, e totalmente reversível a todos os sujeitos que, no final do experimento, usavam o pronome reforçado em todas as frases. Esses resultados evidenciam que os efeitos da história de exposição às contingências mostraram-se temporários, pois apareceram em um primeiro momento, mas tenderam a não se manter quando o comportamento passou a ser punido ou a gerar perda de reforçadores. Além disso, mostraram que a exposição a estímulos apetitivos incontroláveis não necessariamente prejudicou o controle subsequente pela contingência de reforço positivo, achado que se alinha a determinados estudos sobre o desamparo aprendido (Beatty \& Maki, 1979; Calef et al., 1984; Capelari \& Hunziker, 2005).

Quanto aos participantes expostos à história de perdas incontroláveis de pontos, apenas um deles mostrou desempenho semelhante ao dos sujeitos expostos a ganhos incontroláveis de pontos. Em claro contraste, os outros dois sujeitos reduziram e mantiveram baixas as frequências de uso do pronome sob reforço positivo ao longo de todas as 48 frases. Interessantemente, dos dez participantes do estudo, esses foram os dois únicos cuja escolha do pronome "ele" não mostrou efeitos do reforço (i.e., não aumentou em relação à fase anterior) por ganho de pontos.

Esse dado sugere que a perda de pontos, na primeira fase do estudo, de fato funcionou como estímulo aversivo, uma vez que influenciou a aprendizagem posterior, dificultando o controle pelas contingências de reforço positivo. Esse efeito parece ser análogo ao obtido com o modelo clássico de desamparo aprendido (Maier \& Selligman, 1976), com a diferença de que, no presente experimento, essa dificuldade de aprendizagem ocorreu em contingências de reforço positivo, quando, normalmente, sua ocorrência é descrita em contingências de reforço negativo, em tarefas de fuga ou de esquiva.

Dois aspectos desses dados merecem destaque.

342 O fato de a perda de pontos ter atuado como um estí- mulo aversivo faz dela uma alternativa eticamente aceitável em estudos com humanos, contribuindo metodologicamente para estudos futuros sobre o desamparo aprendido. Além disso, o fato de a incontrolabilidade ter afetado uma nova aprendizagem sob contingências de reforço positivo vai ao encontro de dados presentes na literatura (Calef et al., 1986; Caspy \& Lubow, 1981; Rosellini, 1978; Rosellini \& DeCola, 1981; Rosellini et al., 1982), expandindo as ocasiões em que a noção de desamparo aprendido pode ser aplicada. Em especial, trata-se do primeiro estudo que analisa um modelo potencialmente análogo ao desamparo aprendido aplicado a respostas verbais (Skinner, 1957), replicando e generalizando seus achados fundamentais.

Conclusivamente, portanto, os resultados do presente trabalho sugerem que a exposição à incontrolabilidade pode dificultar condições posteriores de novas aprendizagens. No entanto, há que se diferenciar a incontrolabilidade de eventos apetitivos e aversivos. No primeiro caso, os prejuízos, no presente estudo, foram relativamente curtos, concentrados no início do processo e recuperáveis ao longo da exposição às contingências de reforço positivo. No segundo caso, os prejuízos, quando ocorreram, tenderam a prejudicar a nova aprendizagem. Obviamente, essas conclusões são limitadas às condições experimentais presentes e precisam passar pelo escrutínio de manipulações experimentais futuras. No entanto, ainda assim, não deixam de mostrar dados sistemáticos que poderão contribuir aos estudos de incontrolabilidade e desamparo aprendido.

\section{Referências}

Beatty W. W., \& Maki, W. S. (1979). Acquisition of instrumental responding following non-contingent reinforcement: failure to observe "learned laziness" in rats. Bulletin of the Psychonomic Society, 13 (4), 268-271.

Brown, G. E., Howe, A. R., \& Jones, T. E. (1990). Immunization against learned helplessness in the cockroach (Periplaneta americana). Psychological Reports, 67 (2), 635-640.

Calef, R. S., Choban, M. C., Shaver, J. P., Dye, J. D., \& Geller, E. S. (1986). The effect of inescapable shock on the retention of a previously learned response in an appetitive situation with delay of reinforcement. Bulletin of the Psychonomic Society, 24 (3), 213-216.

Calef, R. S., Metz, R. A., Atkinson, T. L., Pellerzi, R. C., Taylor, K. S., \& Geller, E. S. (1984). Acquisition of running in straight alley following experience with response-independent food. Bulletin of the Psychonomic Society, 22 (11), 67-69. 
Capelari, A., \& Hunziker, M. H. L. (2005). Aprendizagem de fuga após estímulos apetitivos incontroláveis. Psicologia: Teoria e Pesquisa, 21 (1), 99-107.

Carvalho Neto, M. B., Maestri, T. C., \& Menezes, E. S. R. (2007). $\mathrm{O}$ jato de ar quente como estímulo aversivo: efeitos de sua exposição prolongada em Rattus norvegicus. Acta Comportamentalia, 15 (2), 171-190.

Caspy T., \& Lubow, R. E. (1981). Generality of US pre-exposure effects: transfer from food to shock or shock to food with and without the same response requirements. Animal Learning and Behavior, 9 (4), 524-532.

Catania, A. C. (1999). Aprendizagem: comportamento, linguagem e cognição. Porto Alegre: Artes Médicas.

Chen, J., \& Amsel, A. (1977). Prolonged, unsignaled, inescapable shocks increase persistance in subsequent appetitive instrumental learning. Animal Learning and Behavior, 5 (4), 377-385.

Enberg, L. A., Welker, R. L., Hansen, G., \& Thomas, D. R. (1972). Acquisition of key pecking via auto shaping as a function to prior experience: "learned laziness"? Science, 178 (4064), 1002-1004.

Ferrándiz, P., \& Vicente, F. (1997). The conditioned attention theory and bifactorial theory on the learned helplessness syndrome in appetitive contexts. International Journal of Psychology, 32 (6), 399-408.

Job, R. F. S. (1987). Learned helplessness in an appetitive discrete trial T-maze discrimination test. Animal Learning and Behavior, 15 (3), 342-346.

Hamasaki, E. I. M., \& Tomanari, G. Y. (2007). Efeitos de diferentes pares de contingências sobre o uso de pronomes na construção de frases. Encontro, 11 (15), 31-51.

Hamasaki, E. I. M., \& Tomanari, G. Y. (2009). Efeitos de diferentes contingências sobre o uso de tempos verbais na construção de frases. Revista Brasileira de Terapia Comportamental e Cognitiva, 11 (1), 119-131.

Hiroto, D. S., \& Seligman, M. E. P. (1975). Generality of learned helplessness in man. Journal of Personality and Social Psychology, 31 (2), 311-327.

Hunziker, M. H. L. (2003). Desamparo aprendido. Tese de livre docência não-publicada, Instituto de Psicologia, Universidade de São Paulo.

Maier, S. F., Albin, R. W., \& Testa, T. J. (1973). Failure to learn to escape in rats previously exposed to inescapable shock depends on nature of escape response. Journal of Comparative and Physiological Psychology, 85 (3), 581-592.

Maier, S. F., \& Seligman, M. E. P. (1976). Learned helplessness: theory and evidence. Journal of Experimental Psychology: General, 105 (1), 3-46.

Matos, M. A., Cirino, S., Passos, M. L., Damiani, K., \& Frochtengarten, F. (1995). O comportamento verbal como operante: uma experiência didática. Resumos da XXV Reunião Anual de Psicologia (p.461). Ribeirão Preto: SBP.
Matos, M. A., \& Tomanari, G. Y. (2002). A análise do comportamento no laboratório didático. São Paulo: Manole.

Matute, H. (1994). Learned helplessness and superstitious behavior as oppositive effects of uncontrollable reinforcement in humans. Learning and Motivation, 25 (2), 216-232.

Overmier, J. B., \& Seligman, M. E. P. (1967). Effects of inescapable shockupon subsequent escape and avoidance responding. Journal of Comparative and Physiological Psychology, 63 (1), 28-33.

Peterson, C., Maier, S. F., \& Seligman, M. E. P. (1993). Learned helplessness: a theory for the age of personal control. New York: Oxford University Press.

Rosellini, R. A. (1978). Inescapable shock interferes with the acquisition of an appetitive operant. Animal Learning and Behavior, 6 (2), 155-159.

Rosellini, R. A., \& DeCola, J. P. (1981). Inescapable shock interferes with the acquisition of a low-activity response in an appetitive context. Animal Learning and Behavior, 9 (4), 487-490.

Rosellini, R. A., DeCola, J. P., \& Shapiro, N. R. (1982). Crossmotivational effects of inescapable shock are associative in nature. Journal of Experimental Psychology: Animal Behavior Process, 8 (4), 376-388.

Seligman, M. E. P., Rosellini, R. A., \& Kozak, M. J. (1975). Learned helplessness in the rat: time course, immunization, and reversibility. Journal of Comparative and Physiological Psychology, 88 (2), 542-547.

Skinner, B. F. (1957). Verbal behavior. New York: AppletonCentury-Crofts.

Sonoda, A., \& Hirai, H. (1992). The role of predictability in preventing escapes deficits following loss of control over. Animal Learning and Behavior, 20 (4), 427-430.

Tomanari, G. Y., Carvalho, A., Góes, Z., Lira, S. B., \& Viana, A. C. V. (2007). Pesquisando ao ensinar: prática no laboratório didático analisa o comportamento verbal sob contingências de reforçamento positivo e negativo. Estudos de Psicologia (Campinas), 24 (2), 205-214. doi: 10.1590/S0103166X2007000200007.

Tomanari, G. Y., Pavão, I. C., \& Benassi, M. T. (2003). Verbal 2.0: um programa de computador para estudos experimentais do comportamento verbal sob controle pelas consequências. In M. Z. S. Brandão, F. C. S. Conte, F. S. Brandão, Y. K. Ingberman, C. B. Moura \& V. M. Silva, et al. (Orgs.), Sobre comportamento e cognição (Vol. 12, pp.379387). Santo André: ESETec.

Welker, R. L. (1976). Acquisition of a free-operant-appetitive response in pigeons as a function of prior experience with response. Learning and Motivation, 7 (3), 394-405.

Wheatley, K. L., Welker, R. L., \& Miles, R. C. (1977). Acquisition of a bar pressing in rats following experience with response independent food. Animal Learning and Behavior, 5 (3), 263-242.

Recebido em: 4/5/2010

Aprovado em: 15/3/2011 
